\title{
Mutational analysis of the early forespore/mother-cell signalling pathway in Bacillus subtilis
}

\author{
J. Arturo Londoño-Vallejo† \\ Tel: +33153725117.e-mail: londono@cephb.fr
}

CNRS UPR-9073, Institut de Biologie Physico-Chimique, Paris, France

\begin{abstract}
Intercellular communication is a crucial phenomenon during spore development in Bacillus subtilis. It couples the establishment of a compartment-specific genetic program to the transcriptional activity of a $\sigma$ factor in the other compartment. It also keeps $\sigma$ factor activation in register with the morphological process. This study used directed mutagenesis to analyse the pathway that couples $\sigma^{E}$ activation in the mother-cell to activation of $\sigma^{\mathrm{F}}$ in the forespore following asymmetric septation. Targets for mutagenesis in SpollGA (the receptor) were chosen based on the predicted topology of the protein when associated with the cell membrane. The results showed that a residue near the $\mathbf{N}$ terminus (D6), predicted to be exposed outside the cell, is required for receptor activity, whereas the major extracellular loop (between membrane domains IV and $V$ ) is dispensable for function. In contrast, mutations in SpollR (the signal) that partially blocked protein release (but not membrane translocation) had no effect on signal transduction. These results do not rule out the possibility that uncharacterized molecules intervene in the signalling pathway that establishes the mother-cell-specific developmental program during the early stage of sporulation.
\end{abstract}

Keywords: Bacillus subtilis, sporulation, signalling pathway, membrane receptor

\section{INTRODUCTION}

Sporulation in Bacillus subtilis is a developmental process initiated in response to nutrient exhaustion and resulting in the production of a dormant, heat-resistant, form: the mature spore. During this process, an ordered sequence of morphological events takes place, starting with the formation of an asymmetrically positioned septum that divides the sporangium into two unequal compartments: the forespore and the mother-cell (Errington, 1993). Each compartment contains a chromosome and engages in a specific genetic program governed by four $\sigma$ factors: $\sigma^{\mathrm{F}}$ and $\sigma^{\mathrm{G}}$ in the forespore, $\sigma^{\mathrm{E}}$ and $\sigma^{\mathrm{K}}$ in the mother-cell. $\sigma$ factor activation, which appears to be co-ordinated with the progression of the morphological stages, is organized in a cascade triggered by activation of $\sigma^{\mathrm{F}}$ and followed by activation of $\sigma^{\mathrm{E}}$, then $\sigma^{\mathrm{G}}$ and finally $\sigma^{\mathrm{K}}$ (Haldenwang, 1995). The molecular events leading to activation of $\sigma$ factors are

†Present address: CEPH, 27 rue Juliette Dodu, Paris 75010, France. Abbreviation: PhoA, alkaline phosphatase. schematically represented in a model called 'criss-cross' (Losick \& Stragier, 1992) in which signals go back and forth through membrane structures between both compartments.

The first event in this scheme links $\sigma^{\mathbf{E}}$ activation in the mother-cell to $\sigma^{\mathrm{F}}$ activity in the forespore. It has been shown recently that transcriptional activity of $\sigma^{\mathrm{F}}$ in the forespore results in the production of SpoIIR (Karow et al., 1995; Londoño-Vallejo \& Stragier, 1995; Zhang et al., 1996) which triggers the conversion of inactive pro$\sigma^{\mathrm{E}}$ into active $\sigma^{\mathrm{E}}$ in the mother-cell. The activation of $\sigma^{\mathbf{E}}$, through cleavage of an N-terminal extension of 28 residues, also requires the presence of SpolIGA, a membrane-associated protein with a large cytoplasmic domain bearing significant similarity to a family of aspartic proteases (Jonas et al., 1988; Masuda et al., 1990; Stragier et al., 1988). Genetic evidence indicates that SpoIIGA itself is the protease responsible for pro- $\sigma^{\mathrm{E}}$ processing during sporulation (Peters \& Haldenwang, 1994). In addition, we have shown, using vegetatively growing cells, that SpoIIR is the only sporulationspecific protein required for activation of SpoIIGA- 
dependent processing of pro- $\sigma^{\mathrm{E}}$ (Londoño-Vallejo $\&$ Stragier, 1995). Moreover, culture supernatants from cells synthesizing SpoIIR are able to trigger production of mature $\sigma^{\mathrm{E}}$ when added extracellularly to nonsporulating whole cells bearing SpoIIGA and pro- $\sigma^{\mathrm{E}}$ (Hofmeister et al., 1995).

Based on these results, current models for the forespore/ mother-cell signalling pathway between $\sigma^{\mathrm{F}}$ and $\sigma^{\mathrm{E}}$ propose two intervening molecules: SpolIR (the signal) and SpoIIGA (the receptor). In an effort to understand the molecular basis of signal-receptor interactions, an analysis of both structural genes using site-directed mutagenesis was undertaken.

\section{METHODS}

In-frame deletions in spollGA. The in-frame deletion in spoIIGA affecting codons $110-132$ was created as follows: an EcoRI-PstI fragment spanning the spollG promoter and the beginning of the gene (up to codon 109) (Guérout-Fleury et al., 1996) was subcloned between the EcoRI site of pUC9 and the PstI site of pUC8, using a third enzyme $(A l w \mathrm{NI})$ to reconstruct the vector. This introduced four codons downstream from the PstI site, a SmaI site compatible and in-frame with the Pvull site in codon 132 of spolIGA. The reconstituted version of spollGA was integrated at amyE in the $B$. subtilis chromosome using pDG1662 (Guérout-Fleury et al., 1996). The large deletion affecting spoIIGA at its own locus between codons 27 and 272 has been described by Guérout-Fleury et al. (1996).

PCR-directed mutagenesis. Point mutations in spoIIGA were introduced by PCR as described by Picard et al. (1994). The following oligonucleotides (complementary strand) carrying the desired point mutations (underlined) were synthesized: YA4, CCAAATGACATCTAAGGCCATTTTCACATC; DA6, GCCAAATGACGGCCAAATAGATTTTCAC; and VA7, TAACAGCCAAATCGCGTCTAAATAGATTTTC. The PCR reactions used a $\Delta P s t \mathrm{I}$ derivative of $\mathrm{pDG} 181$ (promoterless spollGA cloned into pUC18; Stragier et al., 1988) which carried the $5^{\prime}$ end of the gene (beyond the start codon). After PCR, the amplified products were used to reconstitute the structural gene through ligation to the spoIIGA 3 ' end derived from a spoIIGA-HA1 construction (kindly provided by N. Frandsen, IBPC, Paris) and carrying the HA1 epitope tag (Field et al., 1988). The tag, inserted at position M120 of SpoIIGA, has the sequence HAMVPTYPYDVPDYASRPGLGTMACM (epitope underlined). The mutated genes, as well as the wild-type, were placed under the control of the spollA promoter (Stragier et al., 1988) and integrated at the amyE locus of a B. subtilis strain carrying a large deletion in spollGA (Guérout-Fleury et al., 1996). Alternatively, they were placed under the control of the inducible promoter spac within the $B$. subtilis replicative vector pDG178 (Stragier et al., 1988) which carries the whole spollG operon.

The point mutation DE6 was introduced by PCR using the synthetic oligonucleotide GCCAAATGACÉTCGAGATAGATTTTCACATC (complementary strand) and a vector carrying the spollG promoter and the beginning of the spollGA gene (described above) as DNA template. The whole gene was reconstituted and integrated at the amyE locus as indicated above.

Point mutations in spollR (GV21 and AM23) were introduced in the same way using the synthetic oligonucleotide (coding strand) CCGGAGCGCTCGTAGTGCTCATGAAAGAAGAGACGGCAC and a subcloned fragment of spoIIR (with or without promoter) as DNA template. The amplified product was used for reconstitution of spoIIR either under the control of its own promoter or under the control of the inducible promoter spac (Yansura \& Henner, 1984). Both constructions were integrated at $t h r C$ in the $B$. subtilis chromosome using pDG1664 (Guérout-Fleury et al., 1996). In addition, the BspHI site overlapping the AM23 mutation allowed us to construct a version of spollR lacking the first 22 codons after several subcloning steps aimed at replacing the Shine-Dalgarno sequence in front of the methionine. This version was also integrated at $t h r C$ in the chromosome under the control of the spollR promoter. Alternatively, it was placed under the control of the spac promoter.

All point mutations introduced by PCR were confirmed by DNA sequencing. In the case of $s p o I I G A(D A 6)$, when the mutated allele was introduced in single copy into a wild-type strain, the presence of the point mutation in the chromosome after transformation was confirmed by PCR and sequencing.

Constructions of phoA (alkaline phosphatase) and lacZ ( $\beta$ galactosidase) fusions to spollGA. phoA fusions to spoI$I G A$ were constructed by cloning a DNA fragment encoding the soluble domain of PhoA (derived from plasmid pPHO7; Gutierrez \& Devedjian, 1989) in-frame behind several promoterless spollGA fragments. These spoIIGA fragments were generated using the natural restriction sites NheI (codon 65), Tsp509I (codon 87), BspHI (codon 120) and StuI (codon 180). A BamHI restriction site was introduced by PCR at codon 36 . The spollGA-phoA fusions were placed under the control of the inducible promoter spac in the plasmid pDG148 (Stragier et al., 1988). lacZ fusions to spollGA codons 36,87 and 180 were also constructed and placed under the control of spac. pho $A$ and lac $Z$ fusions to spollGA(DA6) were derived from wild-type fusions using the unique NheI site. $p h o A$ fusions were introduced in Escherichia coli $\mathrm{DH} 4 \mathrm{~B}\left(p h o A^{-}\right)$and lacZ fusions in E. coli IBPC5311 (lac $Z^{-}$). PhoA and $\beta$-galactosidase activities were determined in whole cells as described by Brickman \& Beckwith (1975) and Miller (1972). Accumulation of PhoA protein fusions was determined by Western blotting using specific anti-PhoA rabbit antibodies $\left(20 \mu \mathrm{g} \mathrm{ml}^{-1}\right)$ (Rockland). Protein extracts from induced $E$. coli cultures were subjected to SDS-PAGE $(8 \%)$ followed by electrotransfer onto nitrocellulose. After overnight incubation $\left(4^{\circ} \mathrm{C}\right)$, antibodies were revealed by anti-rabbit IgG antibodies conjugated to PhoA (1:5000 dilution) (Promega).

SpollR-levansucrase hybrid proteins. SpoIIR-levansucrase hybrid proteins were constructed by fusing a $R s a \mathrm{I}-B c l \mathrm{I}$ DNA fragment from spollR (spanning the promoter and the first 40 codons) in-frame with a modified $s a c B$ gene (encoding the soluble form of levansucrase; a gift from M. F. Petit-Glatron, Institut Jacques Monod, Paris). The spoIIR fragment encoded either the wild-type or the mutated SpoIIR N-terminal signal sequence. The hybrids were integrated at an ectopic site $(t h r C)$ in the B. subtilis chromosome. All experiments involving levansucrase were performed in strains carrying a disruption in the structural gene $s a c B$. The disruption was introduced in the $B$. subtilis chromosome by marker replacement using a tetracycline resistance cassette (Guérout-Fleury et al., 1995) cloned between sites Asp718 (codon 354) and SacII (codon 360).

Sporulation assays. All $B$. subtilis strains used are derivatives of JH642, except for the protein secretion experiments 
described below. The deletion-disruption in spollR has been described previously (Londoño-Vallejo \& Stragier, 1995). Cells were allowed to sporulate by nutrient exhaustion in liquid DS medium at $37^{\circ} \mathrm{C}$ (Schaeffer et al., 1965). Samples were withdrawn at different times during sporulation and analysed for $\beta$-galactosidase activity or pro- $\sigma^{\mathrm{E}}$ processing as described by Frandsen \& Stragier (1995) and Londoño-Vallejo \& Stragier (1995). Heat-resistant $\left(80^{\circ} \mathrm{C}, 10 \mathrm{~min}\right)$ spores were counted after $30 \mathrm{~h}$ of culture in sporulating medium.

Induction of gene expression under non-sporulating conditions. Conditions of induction of the spac promoter have been described by Frandsen \& Stragier (1995). In experiments including spac-spoIIG and spac-spollR, B. subtilis cell cultures were induced early during exponential growth and samples were withdrawn at different times for $\beta$-galactosidase determination or $\sigma^{\mathrm{E}}$ immunodetection, as described previously (Londoño-Vallejo \& Stragier, 1995). Immunodetection of SpolIGA-HA1 was performed in samples withdrawn $3 \mathrm{~h}$ after induction. Cell lysates were prepared by osmotic shock (Breitling \& Dubnau, 1990) and membrane and soluble fractions, obtained through differential centrifugation, were subjected to SDS-PAGE (12\%) followed by electrotransfer onto nitrocellulose. Blots were incubated with the anti-HA1 monoclonal antibody $\left(5 \mu \mathrm{g} \mathrm{ml}^{-1}, 4^{\circ} \mathrm{C}\right.$, overnight) (Boehringer) and the anti-mouse secondary antibody conjugated to PhoA (1:5000 dilution) (Hyclone).

Detection of SpollR-levansucrase hybrids during sporulation and exponential growth. SpoIIR-levansucrase protein hybrids were detected using purified anti-levansucrase antibodies. The antibodies were affinity-purified using purified levansucrase and a rabbit anti-levansucrase serum (both kindly provided by M.F. Petit-Glatron) as follows. Nitrocellulose-bound levansucrase $(\sim 100 \mu \mathrm{g})$ was incubated with the anti-levansucrase antibodies $\left(1: 20\right.$ dilution) at $4{ }^{\circ} \mathrm{C}$ overnight with continuous shaking. Following extensive washing with TBS $(10 \mathrm{mM}$ Tris, $\mathrm{pH} 8,150 \mathrm{mM} \mathrm{NaCl})$ the antibodies were eluted by treatment with glycine $(0 \cdot 2 \mathrm{M}$, $\mathrm{pH} \mathrm{2.8)} \mathrm{for} 10 \mathrm{~min}$, after which the solution was neutralized with Tris base $(40 \mathrm{mM})$ and equilibrated with TBS.

Protein extracts to be analysed by Western blotting were prepared either from sporulating cells or after induction during vegetative growth. For studies during sporulation, spollR-sacB constructions were introduced into a spoIlAC561 strain carrying a deletion-disruption in $s a c B$. Cells were allowed to sporulate in DS medium and samples were withdrawn at different times. For studies during vegetative growth, spoIIR-sacB constructions were introduced into the protease-deficient strain GP283 (Hofmeister et al., 1995), which carried a plasmid containing the gene for $\sigma^{\mathrm{F}}$ under the control of spac (Londoño-Vallejo \& Stragier, 1995). Induction by addition of IPTG was performed as above and samples were processed after $2 \mathrm{~h}$. Membrane fractions were prepared by differential centrifugation of cell lysates (Breitling \& Dubnau, 1990) and were analysed by SDS-PAGE (10\%) followed by electrotransfer onto nitrocellulose. Supernatants from IPTG-induced cell cultures were filtered $(0.2 \mu \mathrm{m})$ and concentrated using Centriplus10 (Amicon), as described by (Hofmeister et al. (1995). Affinity-purified anti-levansucrase antibodies were used for immunodetection at 1:10 dilution $\left(4{ }^{\circ} \mathrm{C}\right.$, overnight $)$ followed by incubation with an anti-rabbit secondary antibody (1:5000 dilution) coupled to PhoA (Promega).

\section{RESULTS}

\section{Membrane topology of SpollGA and prediction of potential sensor domains}

Computer analysis of the SpolIGA amino acid sequence has predicted the presence of five membrane-spanning domains in the $\mathrm{N}$-terminal half of the protein (Stragier $e t$ al., 1988). The distribution of positive charges found in the connecting loops also predicted that the large soluble $\mathrm{C}$-terminal domain is exposed to the cytosol and that the $\mathrm{N}$-terminus of the protein and two loops (between domains II and III, and IV and V) are found outside the cell (Stragier et al., 1988) (Fig. 1). Indirect evidence for the cytoplasmic localization of the C-terminal domain has been provided by Peters \& Haldenwang (1991) through the construction of a translational lac $Z$ fusion to the $3^{\prime}$ end of the gene, yielding an active $\beta$ galactosidase fusion in E. coli. More evidence in support of the proposed membrane topology for SpoIIGA has been obtained through the construction of five phoA translational fusions at the positions indicated in Table 1. Two of these fusions (positions L65 and M120) yielded enzymically active PhoA fusions in E. coli, indicating their extracellular localization. pho $A$ translational fusions to three other positions (L36, N87 and G180) yielded inactive products, whereas the corresponding lac $Z$ fusions resulted in high $\beta$-galactosidase activity (Table 1 ).

Since SpolIGA activity is controlled through an extracellular signal (and assuming that the topology of the protein in E. coli can be extrapolated to B. subtilis cells) segments exposed on the outer face of the membrane constitute potential sensor domains. In the predicted

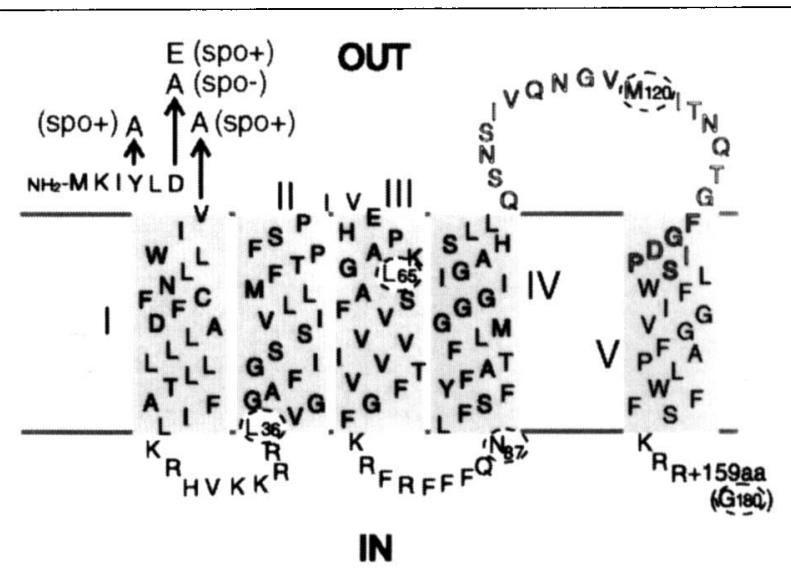

Fig. 1. Schematic representation of the proposed membraneassociated structure of SpollGA, based on sequence and fusion analysis using phoAllacZ. Transmembrane domains are numbered I-V and their residues are represented in a helical net. The C-terminal cytoplasmic domain is not shown. The amino acid positions fused to phoA or lacZ are numbered and indicated by dashed circles. Outlined characters between domains IV and $V$ designate residues dispensable for function. Amino acid replacements affecting positions that are conserved in C. acetobutylicum and their effects on sporulation (spo+, spo-) are indicated by arrows. 
Table 1. PhoA and $\beta$-galactosidase activities obtained from PhoA and $\beta$-galactosidase fusions in SpollGA

Several PhoA and $\beta$-galactosidase fusions were constructed at the indicated amino acid positions in SpoIIGA (ND, not done). The enzymic activities were determined $4-5 \mathrm{~h}$ after the addition of IPTG to exponentially growing E. coli cells carrying the respective constructions. The results of a typical experiment are shown.

\begin{tabular}{|lcc|}
\hline Amino acid & $\begin{array}{c}\boldsymbol{\beta} \text {-Galacto- } \\
\text { sidase } \\
\text { activity } \\
\text { (Miller units) }\end{array}$ & $\begin{array}{c}\text { (Miller units) } \\
\text { L36 }\end{array}$ \\
L65 & 13 & 1375 \\
N87 & 210 & ND \\
M120 & 5 & 1158 \\
G180 & 287 & ND \\
\hline
\end{tabular}

topology, the extracellular loop between membrane domain 2 and 3 appears to be extremely short; therefore, only the larger extracellular loop (between domains IV and $V$ ) and the $\mathrm{N}$-terminal end of the protein were defined as targets for directed mutagenesis (Fig. 1).

A copy of spoIIGA carrying the in-frame deletion $\Delta Q 110-S 132$, which affects the whole extracellular loop between domains IV and V (Fig. 1) and replaces it by the sequence MSRG, was introduced at an ectopic site $(a m y E)$ in the chromosome. This deleted copy fully complemented $\left(\sim 3 \times 10^{8}\right.$ spores $\left.\mathrm{ml}^{-1}\right)$ a large in-frame deletion affecting spoIIGA at its own locus (no spores) (Guérout-Fleury et al., 1996). This result clearly indicates that the big extracellular loop in SpoIIGA is not required to sense the activating signal or, presumably, to transduce it to the catalytic domain inside the cell.

The N-terminal end of SpolIGA, predicted to contain only 6 or 7 aa, stands out by the conservation of some of these residues (Y4, D6, V7) in the SpoIIGA homologue found in the sporulating bacterium Clostridium acetobutylicum (Wong et al., 1995). The predicted overall structure and membrane topology of this protein are very similar to the ones proposed for $B$. subtilis SpoIIGA: five transmembrane domains at their $\mathrm{N}$ terminal half followed by a cytoplasmic domain containing highly conserved amino acid stretches forming the presumed catalytic site (not shown). However, the extracellular connecting loops are less well conserved, underscoring the potential importance of the $\mathrm{N}$-terminal residues. Alanine replacements at positions Y4, D6 and V7 were introduced through PCR mutagenesis and the respective alleles were tested for their ability to complement the $\triangle$ spolIGA strain. YA4 and VA7 mutated genes showed full capacity to complement the sporulation defect whereas the DA6 allele showed a degree of
Table 2. PhoA and $\beta$-galactosidase activities obtained from PhoA and $\beta$-galactosidase fusions in the SpollGA(DA6) mutated protein

PhoA and $\beta$-Galactosidase fusions were constructed at the indicated positions in the SpoIIGA(DA6) mutated protein (DA6) and compared to the activities of the respective wildtype fusions (WT) under the same experimental conditions. ND, Not done.

\begin{tabular}{|cccc|}
\hline Amino acid & Fusion protein & $\begin{array}{c}\text { PhoA activity } \\
\text { (Miller units) }\end{array}$ & $\begin{array}{c}\boldsymbol{\beta} \text {-Galactosidase } \\
\text { activity } \\
\text { (Miller units) }\end{array}$ \\
\hline \multirow{2}{*}{ M120 } & WT & 113 & ND \\
& DA6 & 143 & ND \\
G180 & WT & 8 & 23456 \\
& DA6 & 16 & 25185 \\
\hline
\end{tabular}

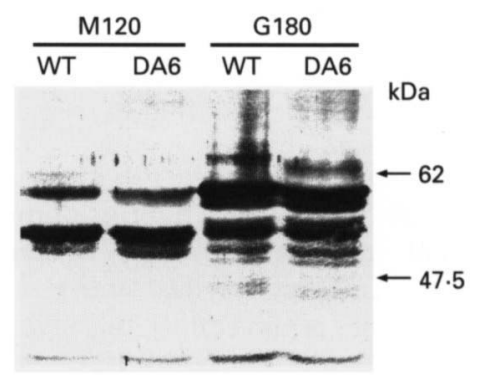

Fig. 2. PhoA fusions to wild-type (WT) and SpollGA(DA6) (DA6). $A$ Western blot analysis using specific antibodies against PhoA shows the relative accumulation of PhoA fusion proteins in cell extracts from the $E$. coli strains assayed in Table 2; the top bands in each lane correspond to the expected full-length fusion proteins.

complementation two to three orders of magnitude below the normal level $\left(3 \times 10^{5}-1 \times 10^{6}\right.$ spores $\left.\mathrm{ml}^{-1}\right)$. The N-terminal DA6 point mutation apparently did not affect the overall SpoIIGA protein structure in the membrane since PhoA or $\beta$-galactosidase fusions to SpoIIGA(DA6) behaved identically to the wild-type fusions in E. coli (Table 2 and Fig. 2). No dominant negative effect on sporulation was observed when the spoIIGA(DA6) allele was introduced in an otherwise wild-type strain, the presence in the chromosome of the mutated allele being confirmed by PCR and sequencing. Interestingly, a DE6-mutated spoIIGA allele was able to fully complement the sporulation defect of the $\triangle$ spoIIGA strain when introduced at an ectopic site $(a m y E)$.

\section{The N-terminal domain of SpollGA is essential for SpollGA-dependent $\sigma^{\mathrm{E}}$ activation}

Experiments were performed to follow the kinetics of pro- $\sigma^{\mathrm{E}}$ processing and of transcription of $\sigma^{\mathrm{E}}$-dependent 

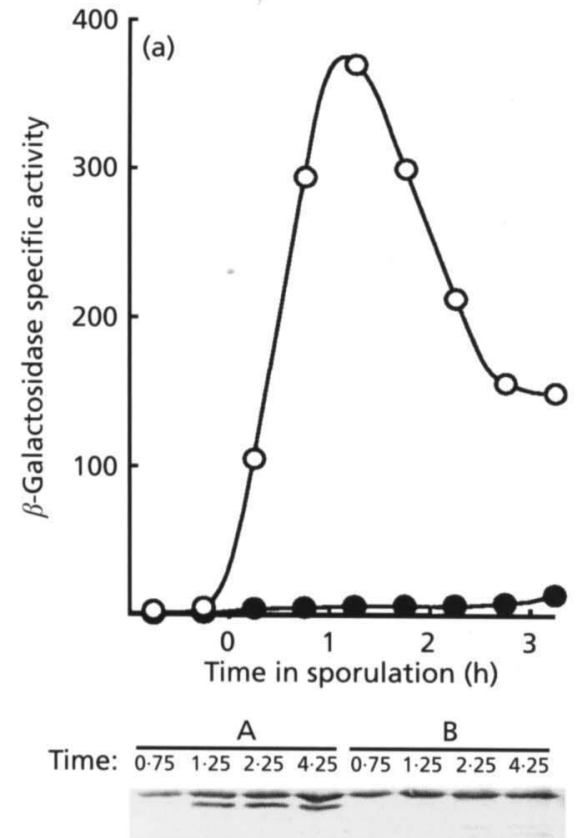

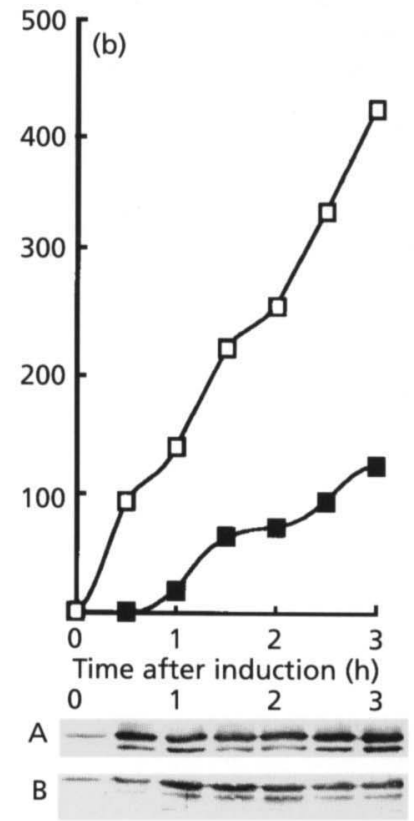

(c)

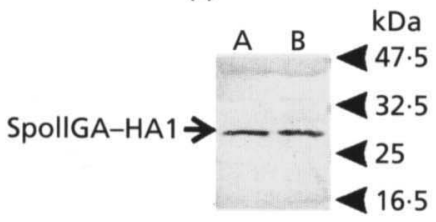

Fig. 3. Effects of the DA6 mutation in SpollGA function. (a) Time course of expression during sporulation of a spollD-lacZ transcriptional fusion (dependent on $\sigma^{\mathrm{E}}$ ) in a $\triangle$ spol/GA strain carrying either spol/GA-HA1 (O, top; $A$, lower) or spo//GA(DAG)-HA1 at the amyE locus (O, top; B, lower). The lower part shows an immunoblot analysis of pro- $\sigma^{E}$ processing in the same set of strains, samples being harvested at the times indicated (h). (b) Time course of expression of the spollD-lacZ transcriptional fusion in strains engineered to produce pro- $\sigma^{E}$, SpollR and either SpollGA-HA1 ( $\square$, top; A, lower) or SpollGA(DA6)-HA1 ( $\square$, top; B, lower) in response to IPTG. The lower part shows an immunoblot analysis of pro- $\sigma^{E}$ processing in the same set of strains and samples. (c) SpollGA immunoblot analysis, using anti-HA1 monoclonal antibody and membrane protein extracts from cells harvested at the last time point in (b). Lanes: A, SpollGA-HA1; B, SpollGA(DA6)HA1. Approximate mobility: $30 \mathrm{kDa}$ (expected: $37.5 \mathrm{kDa}$ ). $\beta$-Galactosidase activity is expressed as nmol 2-nitrophenyl- $\beta$-D-galactopyranoside hydrolysed $\mathrm{min}^{-1}(\mathrm{mg} \text { protein })^{-1}$. genes during sporulation in the $\triangle$ spoIIGA strain carrying a spoIIGA(DA6) allele. In the typical experiment shown in Fig. $3(\mathrm{a})$, pro- $\sigma^{\mathrm{E}}$ processing was undetectable and expression of spoIID-lacZ (a member of the $\sigma^{\mathrm{E}}$ regulon; Rong et al., 1986) was highly depressed when compared to the control strain.

The wild-type and DA6 spollGA versions used in this experiment carried the epitope tag HA1 (Field et al., 1988) at position M120, and both versions were placed under the control of a relatively strong stage 0 promoter (spollA) (Errington \& Mandelstam, 1986; Wu et al., 1991). However, both proteins were undetectable in Western blots using the anti-HA1 monoclonal antibody (not shown); this suggested a very low level of expression of this particular gene during sporulation.

To circumvent this problem, a system was used in which spoIIG (encoding both SpoIIGA and pro- $\sigma^{\mathrm{E}}$ ) as well as spoIIR are controlled by an inducible promoter (Londoño-Vallejo \& Stragier, 1995; Stragier et al., 1988). Induction of these genes during vegetative growth (non-sporulation conditions) results in pro- $\sigma^{\mathrm{E}}$ processing and transcription of $\sigma^{\mathrm{E}}$-dependent genes (Londoño-Vallejo \& Stragier, 1995). After induction, the strain bearing the mutated version of spoIIGA-HA1 showed impaired $\sigma^{\mathrm{E}}$ processing and consequently low $\sigma^{\mathrm{E}}$ transcriptional activity when compared to the control strain (Fig. 3b). The residual activity observed for SpoIIGA(DA6) was still dependent on the presence of
spoIIR (not shown). Western blot analysis showed that both wild-type and SpoIIGA(DA6) proteins accumulated in cell-membrane fractions and were undetectable in the cytosol (not shown). Although, as shown in Fig. 3 (c), SpoIIGA(DA6) seemed to accumulate at a slightly lower level than the wild-type, protein instability would not fully account for the fourfold difference between the $\sigma^{\mathrm{E}}$ transcriptional activities detected in the same strains (Fig. 3b).

\section{Release of SpollR is not necessary for SpollGA activation}

Analysis of the SpolIR amino acid sequence predicted that the protein is exported through the membrane and secreted due to a typical $\mathrm{N}$-terminal signal peptide (Karow et al., 1995). Those predictions were confirmed by the finding that a protein factor possessing the characteristics of SpoIIR is released in the culture supernatants of cells induced for $\sigma^{\mathbf{F}}$ activity (Hofmeister et al., 1995). The SpollR secretion signal peptide contains the highly conserved amino acids glycine and alanine at positions -3 and -1 , respectively, from the presumed cleavage point (Nagarajan, 1993).

To test whether release from the membrane is necessary for SpoIIR activity, point mutations were introduced by PCR at these conserved positions (GV21 and AM23). These mutations were expected to block cleavage but 
(a)

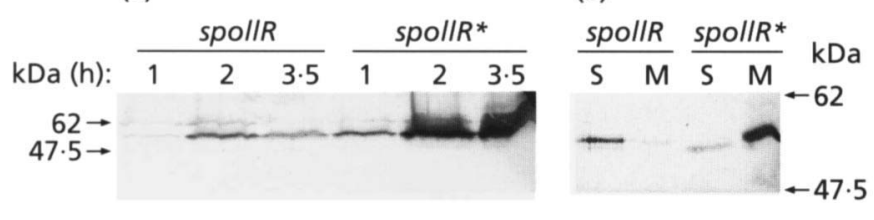

spollAC561 genetic background (Illing \& Errington, 1991). These strains, which produced a modified form of $\sigma^{F}$ resulting in a higher expression of spollR, were allowed to engage in sporulation and cell samples were analysed by Western blotting at different times. As shown in Fig. 4(a), signals corresponding to the SpoIIRlevansucrase hybrids were visible in membrane fractions from both strains. However, only the mutated version accumulated with time, suggesting that its release from the membrane was somewhat impaired by the changes introduced at the putative cleavage site of SpoIIR, whereas most of the wild-type SpoIIR-levansucrase hybrid was presumably released into the medium.

Next, the fate of the hybrid SpoIIR-levansucrase proteins after $\sigma^{\mathbf{F}}$ induction was determined by probing the supernatant and membrane extracts from exponentially growing cell cultures. As shown in Fig. 4(b), the wild-type SpoIIR signal sequence mediated secretion of SpoIIR-levansucrase, which was found almost exclusively in the supernatant, whereas mutations in the SpoIIR putative cleavage site led to accumulation of the hybrid protein in the membrane with a small proportion being detected in solution. Because the observed relative mobilities of the secreted form in the wild-type and the membrane-associated form in the mutant were not different in the gel system used here, it is not known whether protein accumulation in the membrane actually resulted from blockage of cleavage in the signal sequence of SpoIIR.

The mutations introduced in the signal sequence of SpoIIR, although they partially inhibited protein release, do not appear to affect its function, namely the activation of SpoIIGA. This conclusion is reinforced by induction experiments during vegetative growth in which the kinetics and efficiency of this activation is indistinguishable from that observed with the wild-type SpoIIR (Fig. 5a). To further test the possibility that protein release might not be required for SpoIIR signalling, a conventional $\mathrm{N}$-terminal membrane domain, found in the forespore-specific membrane protein SpoIIQ (domain 1-MREEEKKTSQVKKLQQFFRKR WVFPAIYLVSAAVILTAVLWYQSVSNDEVKD-52, with the same orientation and with no putative cleavage site; Londoño-Vallejo et al., 1997), was substituted for the signal peptide in SpoIIR. The spoIIQspoIIR version was able to fully complement $\left(3 \times 10^{8}\right.$ spores $\mathrm{ml}^{-1}$ ) the sporulation defect when integrated at the amyE locus in the B. subtilis chromosome of a $\Delta$ spollR strain. This observation suggests that the SpoIIQ membrane domain mediated translocation of SpolIR through the forespore membrane and further supports the idea that release of SpoIIR within the interseptal space might not be required to activate SpolIGA during sporulation.

Finally, the ability of the SpoIIR mutated proteins to activate SpoIIGA in the same cell compartment was explored. To do this, the spoIIR(GV21-AM23) allele (or the 'cytoplasmic' spoIIR version) was substituted for 


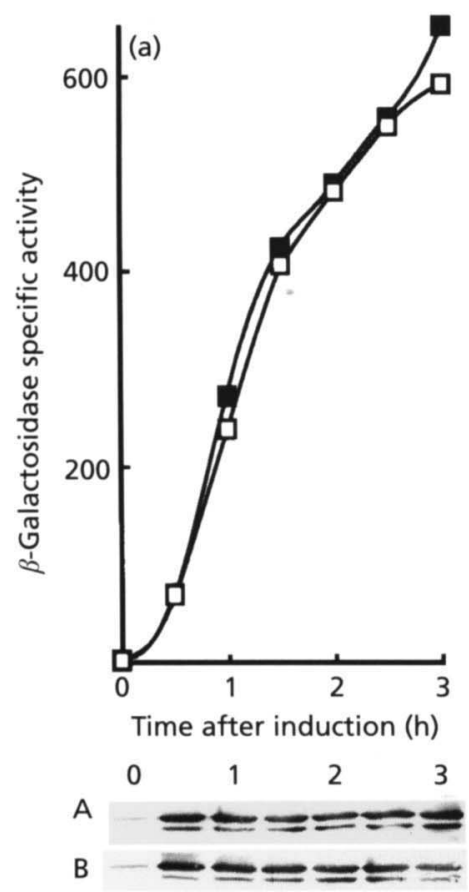

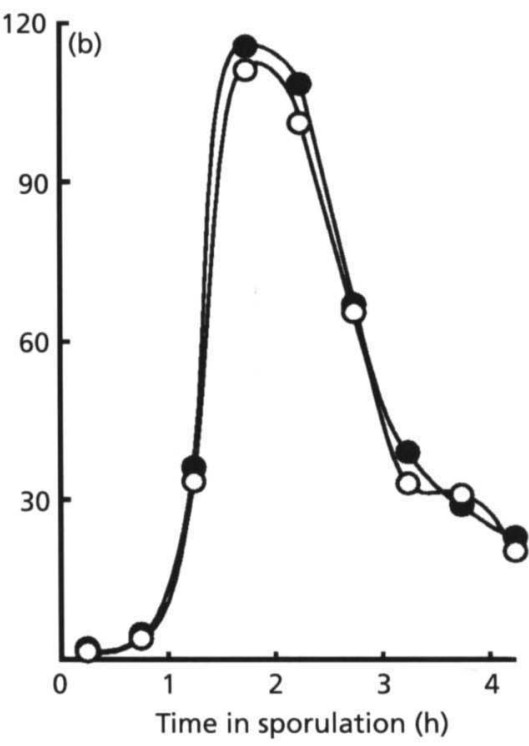

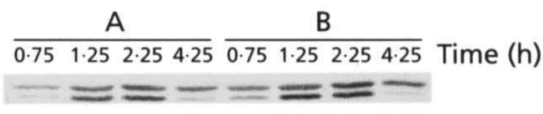

Fig. 5. Efficiency of SpollGA activation by SpollR(GV21-AM23). (a) Time course of expression of the spollD-lacZ transcriptional fusion in strains engineered to produce pro$\sigma^{\mathrm{E}}$, SpollGA and either SpollR ( $\square$, top; A,

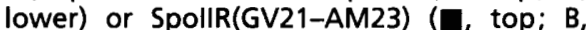
lower) in response to IPTG. The latter strain carried a spollR null mutation in the chromosome. The lower part shows an immunoblot analysis of pro- $\sigma^{\mathrm{E}}$ processing in the same set of strains and samples. (b) Time course of expression during sporulation of a spollD-lacZ transcriptional fusion in $\mathrm{MO}$ 1300 strains carrying either a wild-type spollR allele at its own locus $(O$, top; $A$, lower) or a null mutation in spollR and the allele SpollR(GV21-AM23) at an ectopic site (O, top; B, lower). The lower part shows an immunoblot analysis of pro- $\sigma^{\mathrm{E}}$ processing in the same set of strains, samples being harvested at the times indicated (h). $\beta$-Galactosidase activity is expressed as nmol 2-nitrophenyl- $\beta$-D-galactopyranoside hydrolysed $\operatorname{~min}^{-1}$ (mg protein) $)^{-1}$. the wild-type allele in the $B$. subtilis strain MO1300 (P"spoll $A-\Delta$ spoll $A B)$. In this strain, SpolIAB, the anti- $\sigma$ factor that prevents $\sigma^{F}$ activation before septation (Duncan \& Losick, 1993), is absent; this allows $\sigma^{\mathrm{F}}$ activity to appear before septation, a second mutation in the spollA promoter keeping active $\sigma^{F}$ levels low enough to prevent immediate cell lysis (Shazand et al., 1995). Nevertheless, active $\sigma^{\mathrm{F}}$ triggers activation of $\sigma^{\mathrm{E}}$, which leads to definitive blockage of asymmetric septation (Shazand et al., 1995). Since activation of $\sigma^{\mathrm{E}}$ in MO1300 is not due to a long-range intercellular signal (LondoñoVallejo \& Stragier, 1995), it is better explained by the ability of SpoIIR to activate SpoIIGA molecules inserted on the same membrane surface from which the signal is released. As shown in Fig. 5(b), SpoIIR(GV21-AM23) is as efficient as the wild-type protein in triggering pro- $\sigma^{\mathrm{E}}$ processing by SpoIIGA in non-compartmentalized cells. No pro- $\sigma^{\mathbf{E}}$ processing was detected in the MO1300 genetic context when the 'cytoplasmic' version of SpoIIR was used to replace the wild-type spoIIR gene (not shown), suggesting that membrane translocation does favour the interaction between signal and receptor.

\section{DISCUSSION}

A genetic approach to investigate the presumed interactions between the forespore signal (SpoIIR) and the mother-cell receptor (SpoIIGA) was followed. Based on protein sequence analysis and experimental evidence, mutations in two extracellularly exposed domains of SpoIIGA were designed. The results showed that the large extracellular loop between membrane domains IV and $\mathrm{V}$ is dispensable for function. On the other hand, an aspartate at position 6 appears to be important for triggering pro- $\sigma^{\mathrm{E}}$ proteolytic activation. However, the reasons for the loss of function in the SpoIIGA(DA6) protein remain obscure.

The induction experiments during vegetative growth suggested that impairment in $\sigma^{\mathrm{E}}$ activation by SpoIIGA(DA6) is not caused by protein instability or by interference with the correct targeting of the protein to a specific location in the cell (for example, the sporulation septum). Interestingly, a more conservative amino acid change (to glutamate) preserves the function of the protein. Perhaps this negatively charged residue is part of a surface which contacts either SpoIIR itself or another protein involved in signal transduction.

This report also presents evidence suggesting that membrane translocation of SpoIIR is required for full SpoIIGA activation. On the other hand, mutations in the putative cleavage site of SpoIIR that partially block release of the fusion protein SpoIIR-levansucrase do not affect protein function. In this case, the release of small amounts of SpoIIR could theoretically be enough to activate SpoIIGA (Hofmeister et al.; 1995). However, the replacement of the whole signal sequence in SpoIIR by a conventional membrane domain did not affect its function either, suggesting that membrane-exported SpoIIR may not need to be released to activate SpoIIGA.

Clearly, more powerful experiments are required to demonstrate a direct interaction between SpoIIR and SpolIGA or else the participation of intermediate molecules. An in vitro reconstitution of the system would provide the best tool to test the relevance and topology of partner interactions as well as to understand the molecular mechanisms leading to receptor activation. Alternatively, directed mutagenesis aimed at 
modifying the properties of the SpoIIGA transmembrane domains could shed light on the way signal transduction takes place during the early stages of spore development.

\section{ACKNOWLEDGEMENTS}

This work was carried out in the laboratory of P. Stragier. I thank all the members of his laboratory for their support. Thanks to A.-M. Guérout, N. Frandsen and M.-F. Petit-Glatron for some of the material used in this study. Thanks also to A. Hofmeister, D. Dubnau and two anonymous reviewers for their helpful suggestions.

\section{REFERENCES}

Borchert, T. V. \& Nagarajan, V. (1991). Effect of signal sequence alterations on export of levansucrase in Bacillus subtilis. $J$ Bacteriol 173, 276-282.

Breitling, R. \& Dubnau, D. (1990). A membrane protein with similarity to $\mathrm{N}$-methylphenylalanine pilins is essential for DNA binding by competent Bacillus subtilis. J Bacteriol 172, 1499-1508.

Brickman, E. \& Beckwith, J. (1975). Analysis of the regulation of Escherichia coli alkaline phosphatase synthesis using deletions and $\phi 80$ transducing phages. $J \mathrm{Mol} \mathrm{Biol} \mathrm{96,} \mathrm{307-316.}$

Duncan, L. \& Losick, R. (1993). SpoIIAB is an anti- $\sigma$ factor that binds to and inhibits transcription by regulatory protein $\sigma^{\mathrm{F}}$ from Bacillus subtilis. Proc Natl Acad Sci USA 90, 2325-2329.

Errington, J. (1993). Sporulation in Bacillus subtilis : regulation of gene expression and control of morphogenesis. Microbiol Rev 57, $1-33$.

Errington, J. \& Mandelstam, J. (1986). Use of a lacZ gene fusion to determine the dependence pattern of sporulation operon spollA in spo mutants of Bacillus subtilis. J Gen Microbiol 132, 2967-2976.

Field, J., Nikawa, J.-I., Broek, D., MacDonald, B., Rogers, L., Wilson, I. A., Lerner, R. A. \& Wigler, M. (1988). Purification of a RAS-responsive adenylyl cyclase complex from Saccharomyces cerevisiae by use of an epitope addition method. Mol Cell Biol 8, 2159-2165.

Frandsen, N. \& Stragier, P. (1995). Identification and characterization of the Bacillus subtilis spollP locus. J Bacteriol 177, 716-722.

Guérout-Fleury, A.-M., Shazand, K., Frandsen, N. \& Stragier, P. (1995). Antibiotic-resistance cassettes for Bacillus subtilis. Gene 167, 335-336.

Guérout-Fleury, A.-M., Frandsen, N. \& Stragier, P. (1996). Plasmids for ectopic integration in Bacillus subtilis. Gene 180, 57-61.

Gutierrez, C. \& Devedjian, J.-C. (1989). A plasmid facilitating in vitro construction of $p h o A$ gene fusions in Escherichia coli. Nucleic Acids Res 17, 3999.

Haldenwang, W. G. (1995). The sigma factors of Bacillus subtilis. Microbiol Rev 59, 1-30.

Hofmeister, A. E. M., Londoño-Vallejo, A., Harry, E., Stragier, P. \& Losick, R. (1995). Extracellular signal protein triggering the proteolytic activation of a developmental transcription factor in B. subtilis. Cell 83, 219-226.

Illing, N. \& Errington, J. (1991). Genetic regulation of mor- phogenesis in Bacillus subtilis: roles of $\sigma^{\mathrm{E}}$ and $\sigma^{\mathrm{F}}$ in prespore engulfment. J Bacteriol 173, 3159-3169.

Jonas, R. M., Weaver, E. A., Kenney, T. J., Moran, C. P., Jr \& Haldenwang, W. G. (1988). The Bacillus subtilis spoIIG operon encodes both $\sigma^{\mathrm{E}}$ and a gene necessary for $\sigma^{\mathrm{E}}$ activation. J Bacteriol 170, 507-511.

Karow, M. L., Glaser, P. \& Piggot, P. J. (1995). Identification of a gene, spoIIR, which links the activation of $\sigma^{\mathbf{E}}$ to the transcriptional activity of $\sigma^{F}$ during sporulation in Bacillus subtilis. Proc Natl Acad Sci USA 92, 2012-2016.

Londoño-Vallejo, J.-A. \& Stragier, P. (1995). Cell-cell signaling pathway activating a developmental transcription factor in Bacillus subtilis. Genes Dev 9, 503-508.

Londoño-Vallejo, J.-A., Fréhel, C. \& Stragier, P. (1997). spoIIQ, a forespore expressed gene required for engulfment in Bacillus subtilis. Mol Microbiol 24, 29-39.

Losick, R. \& Stragier, P. (1992). Crisscross regulation of cell-type specific gene expression during development in Bacillus subtilis. Nature 355, 601-604.

Masuda, E. S., Anaguchi, H., Sato, T., Takeuchi, M. \& Kobayashi, Y. (1990). Nucleotide sequence of the sporulation gene spollGA from Bacillus subtilis. Nucleic Acids Res 18, 657.

Miller, J. (1972). Experiments in Molecular Genetics. Cold Spring Harbor, NY: Cold Spring Harbor Laboratory.

Nagarajan, V. (1993). Protein secretion. In Bacillus subtilis and other Gram-positive bacteria: Biochemistry, Physiology and Molecular Genetics, pp 713-726. Edited by A. L. Sonenshein, J. A. Hoch \& R. Losick. Washington, DC: American Society for Microbiology.

Peters, H. K., III \& Haldenwang, W. G. (1991). Synthesis and fractionation properties of spoll $G A$, a protein essential for pro- $\sigma^{\mathrm{E}}$ processing in Bacillus subtilis. J Bacteriol 173, 7821-7827.

Peters, H. K. \& Haldenwang, W. G. (1994). Isolation of a Bacillus subtilis spollGA allele that suppresses processing-negative mutations in the Pro- $\sigma^{\mathrm{F}}$ gene (sigE). J Bacteriol 176, 7763-7766.

Petit-Glatron, M. F., Monteil, I., Benyahai, F. \& Chambert, R. (1990). Bacillus subtilis levansucrase: amino acid substitutions at one site affect secretion efficiency and refolding kinetics mediated by metals. Mol Microbiol 4, 2063-2070.

Picard, V., Ersdal-Badju, E., Lu, A. \& Clark Bock, S. (1994). A rapid and efficient one-tube PCR-based mutagenesis technique using Pfu DNA polymerase. Nucleic Acids Res 22, 2587-2591.

Rong, S., Rosenkrantz, M. S. \& Sonenshein, A. L. (1986). Transcriptional control of the Bacillus subtilis spoIID gene. J Bacteriol 165, 771-779.

Schaeffer, P., Millet, J. \& Auber, J.-P. (1965). Catabolite repression of bacterial sporulation. Proc Natl Acad Sci USA 54, 704-711.

Shazand, K., Frandsen, N. \& Stragier, P. (1995). Cell-type specificity during development in Bacillus subtilis: the molecular and morphological requirements for $\sigma^{\mathrm{E}}$ activation. EMBO J14, 1439-1445.

Stragier, P., Bonamy, C. \& Karmazyn-Campelli, C. (1988). Processing of a sporulation sigma factor in Bacillus subtilis: how morphological structure could control gene expression. Cell 52, 697-704.

Wong, J., Sass, C. \& Bennett, G. N. (1995). Sequence and arrangement of genes encoding sigma factors in Clostridium acetobutylicum ATCC 824. Gene 153, 89-92.

Wu, J.-J., Piggot, P. J., Tatti, K. M. \& Moran, C. P. J. (1991). Transcription of the Bacillus subtilis spollA operon. Gene 101, 113-116. 
Yansura, D. G. \& Henner, D. J. (1984). Use of the Escherichia coli lac repressor and operator to control gene expression in Bacillus subtilis. Proc Natl Acad Sci USA 81, 439-443.

Zhang, L., Higgings, M. L., Piggot, P. J. \& Karow, M. L. (1996).

Analysis of the role of prespore gene expression in the com- partmentalization of mother cell-specific gene expression during sporulation of Bacillus subtilis. J Bacteriol 178, 2813-2817.

Received 3 February 1997; revised 1 April 1997; accepted 7 April 1997. 